\title{
Confirmation of two extended objects along the line of sight to PKS 1830-211 with ESO-VLT adaptive optics imaging ${ }^{\star}$
}

\author{
G. Meylan ${ }^{1}$, F. Courbin ${ }^{1}$, C. Lidman² ${ }^{2}$ J.-P. Kneib ${ }^{3}$, and L. E. Tacconi-Garman ${ }^{4}$ \\ ${ }^{1}$ Laboratoire d'Astrophysique, École Polytechnique Fédérale de Lausanne (EPFL), Observatoire, 1290 Sauverny, Switzerland \\ 2 European Southern Observatory, Casilla 19001, Santiago, Chile \\ ${ }^{3}$ LAM, Observatoire Astronomique de Marseille-Provence, Traverse du Siphon - BP 8, 13376 Marseille Cedex 12, France \\ ${ }^{4}$ European Southern Observatory, Karl-Schwarzschild-Straße 2, 85748 Garching bei München, Germany
}

Received 11 March 2005 / Accepted 22 June 2005

\begin{abstract}
We report on new high-resolution near-infrared images of the gravitationally lensed radio source PKS 1830-211, a quasar at $z=2.507$. These adaptive optics observations, taken with the Very Large Telescope (VLT), are further improved through image deconvolution. They confirm the presence of a second object along the line of sight to the quasar, in addition to the previously known spiral galaxy. This additional object is clearly extended in our images. However, its faint luminosity does not allow to infer any photometric redshift. If this galaxy is located in the foreground of PKS 1830-211, it complicates the modeling of this system and decreases the interest in using PKS 1830-211 as a means to determine $H_{0}$ via the time delay between the two lensed images of the quasar.
\end{abstract}

Key words. gravitational lensing - quasars: individual: PKS 1830-211

\section{Introduction}

PKS 1830-211 is one of the first quasars that was found to be lensed. It is seen in the radio as a full Einstein ring connecting two quasar images (Rao \& Subrahmanyan 1988; Subrahmanyan et al. 1990; Jauncey et al. 1991). The time delay between the quasar components is known from an 18-month radio monitoring with ATCA at $8.6 \mathrm{GHz}$, providing $\Delta t=$ $26 \pm 5$ days (Lovell et al. 1998). While the quasar is bright in the radio, it is much fainter in the optical. It is only in nearinfrared that both quasar images can be identified, either using ESO/KECK I, J, K images (Courbin et al. 1998), or the Hubble Space Telescope (HST) with the NICMOS instrument (Lehár et al. 2000).

The redshift of the background quasar is known from IR spectroscopy, $z=2.507$, using the redshifted $\mathrm{H} \alpha$ emission line (Lidman et al. 1999), while the identification of the lensing galaxy has long remained problematic. Indirect identification of the lens was already possible in early millimeter observations of PKS 1830-211, thanks to strong absorption lines at $z=0.89$ that were found in the spectra of the quasar

* Based on observations obtained at the European Southern Observatory using the Very Large Telescope, Cerro Paranal, Chile (ESO Program ID 71.A-0401(A), PI: G. Meylan). Based in part on data obtained with the NASA/ESA Hubble Space Telescope (Program \#9744, PI: C. S. Kochanek) and extracted from the data archives at the Space Telescope Science Institute, which is operated by the Association of Universities for Research in Astronomy, Inc., under NASA contract NAS 5-26555. images (Wiklind \& Combes 1996). The direct identification of a lensing object, in the form of a spiral galaxy, is much more recent: using HST optical and IR images Courbin et al. (2002, hereafter C02) and Winn et al. (2002, hereafter W02) identify a spiral galaxy between the quasar images. The $V-I$ color of this galaxy indicates that it is probably associated with the $z=0.89$ millimeter absorber.

In addition to the spiral lens found by both $\mathrm{C} 02$ and W02, $\mathrm{C} 02$ also noticed the presence of a second diffuse light source between the quasars, which is already pointed out by Lehár et al. (2000). While Lehár et al. (2000) and C02 identify this object as a lensing galaxy, W02 assume that it is an image artifact or a feature associated with either the quasar or the more obvious lensing spiral galaxy. This second object, "Lens G", is faint and very close to the center of the spiral galaxy and is seen only in the IR (see Fig. 1). Only deep near-infrared imaging with Adaptive Optics (AO) on a 10-m class telescope can significantly improve the quality of the current observations. This paper presents such new near-infrared AO images, obtained with the VLT. The data, further improved with deconvolution techniques, allow us to confirm unambiguously the second object, "Lens G", located between the two quasar images, without, however, being able to put any constraints on its redshift.

\section{VLT adaptive optics observations and reduction}

Deep $J, H$ and $K_{\mathrm{s}}$-band images of PKS 1830-211 were obtained at the ESO Paranal Observatory with the near-infrared 


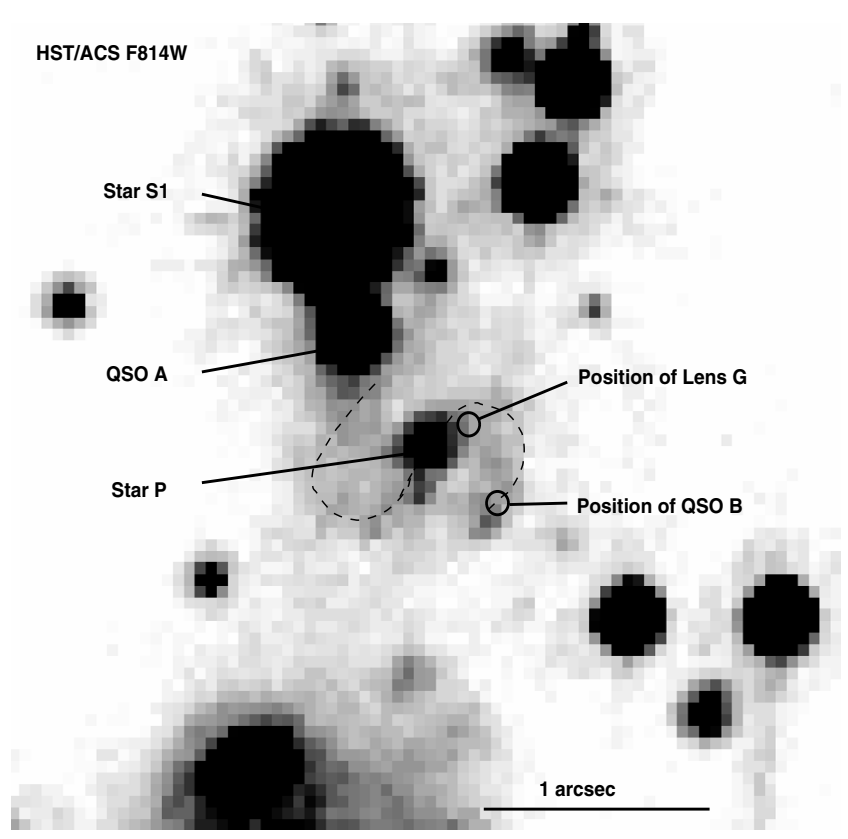

Fig. 1. Part of an HST/ACS F814W image obtained by the CASTLE group. This deep $10390 \mathrm{~s}$ exposure shows the spiral lens (dashed line) pointed out by $\mathrm{C} 02$ and W02. Component A of the quasar is conspicuous while the component $\mathrm{B}$ is obscured by one the spiral arms of the lensing galaxy, which center is not unambiguously identified: W02 consider Star P as the bright unresolved nucleus of the spiral, while $\mathrm{C} 02$ consider it as a mere star. The position of Lens G, confirmed by the present VLT AO observations is indicated. All objects are labeled as in C02. North is up and East to the left.

camera CONICA (the COude Near-Infrared CAmera), which is mounted on the AO system NAOS (Nasmyth Adaptive Optics System). Both CONICA and NAOS are mounted on the Nasmyth focus of VLT UT4 (Yepun). The $J$ and $H$-band data were taken during the night of 27 May, 2003 and the $K_{\mathrm{s}}$-band data were taken during the night of 3rd September, 2003. The pixel size of the instrument, $0{ }^{\prime} 02704 \pm 00^{\prime} 00007$, allows the acquisition of well sampled data at the diffraction limit of the telescope.

Until the laser guide star becomes available at the VLT, a nearby bright star is required to carry out the wavefront correction by the AO system. Thanks to the low galactic latitude of PKS $1830-211$, a $V=13.5$ star is available 7" to the NorthEast of the quasar. It is the same star that was used for the $K$-band Gemini AO observations presented in Fig. 1 of $\mathrm{C} 02$. The seeing during these observations results in a final AOcorrected resolution that is always better than $0 . ' 1$.

Standard reduction techniques are applied to the data. They include dark subtraction, flat fielding using twilight flats, and sky subtraction. The sky frames are computed using the $14 \mathrm{im}-$ ages taken nearest in time to the frame considered. The reduced frames are then registered and combined, rejecting the highest and the lowest pixels in the stack. Cosmic rays and pixels with variable dark current are removed, since dithering is applied during the observations. The details of the observations are summarized in Table 1 .
Table 1. Summary of the VLT adaptive optics observations of PKS 1830-211. The length of the individual exposures is DIT seconds. NDIT exposures are averaged for each of the NDITHER telescope positions. The value of the AO-corrected resolution is the FWHM of the core of the adaptive optics PSF.

\begin{tabular}{lcccr}
\hline \hline Filter & $\begin{array}{c}\text { Airmass } \\
(\text { mean })\end{array}$ & $\begin{array}{c}\text { NDIT } \times \text { DIT } \\
\times \text { NDITHER }\end{array}$ & $\begin{array}{r}\text { Resolution } \\
(\text { FWHM })\end{array}$ & $\begin{array}{r}\text { Exp. time } \\
\text { (total) }\end{array}$ \\
\hline$J$ & 1.10 & $3 \times 40 \times 37$ & $0^{\prime} 085$ & $4440 \mathrm{~s}$ \\
$H$ & 1.20 & $3 \times 20 \times 104$ & $0^{\prime} 070$ & $6240 \mathrm{~s}$ \\
$K \mathrm{~s}$ & 1.03 & $2 \times 30 \times 14$ & $0^{\prime} 097$ & $840 \mathrm{~s}$ \\
\hline
\end{tabular}

\section{Deconvolution and astrometry}

Two objects are striking in the new AO data (Fig. 2, left panels), especially in the $H$ filter: Star P from C02, and Lens G, at the position already pointed out by $\mathrm{C} 02$ and Lehár et al. (2000). The spiral lens seen in the HST optical images is too faint to be detected in these near-infrared images.

While Lens $\mathrm{G}$ is already nicely confirmed by the adaptive optics images, image deconvolution allows one to go further and to derive extremely precise astrometry. The MCS deconvolution algorithm (Magain et al. 1998) is used, reaching a resolution of 0'.027 in all three near-infrared filters. The data are decomposed into a point-source (stellar and quasar images) and extended "channels". The lensing galaxies and the quasar host are represented in this latter channel. We note an extended object in the $H$-band image surrounding the quasar Image B. It can be either the lensed host of the quasar, or one more intervening object along the line of sight. The pixel scale in the deconvolved image has been improved by a factor of two over the original data, i.e. the new pixel size is $0{ }^{\prime} 01352$. The deconvolved $J$ and $H$-band images are displayed in Fig. 2 (right panels). Lens $\mathrm{G}$ is also visible in the shallower $K_{\mathrm{s}}$-band data.

The quality of the deconvolved images is mainly limited by the strong PSF variation across the field of view. As a result, and although several stars are available in order to carry out the PSF calculation within a circle of 10" around PKS 1830-211, small artifacts are seen close to the quasar images at faint levels. This effect is significant only near the bright objects $\mathrm{S} 1$ and quasar Image A and does not affect the deconvolution at the location of the much fainter Lens $\mathrm{G}$. These faint artifacts, shown in log scale in Fig. 2, are a few percent of the peak intensity of Lens G. The PSF wings are modelled over an area which has the same size as the field used for the deconvolution.

We summarize in Table 2 and Fig. 3 the astrometry of the different objects. All objects are visible in the $J, H$ and $K_{\mathrm{s}}$-bands. The quasar Image B is nevertheless very faint in $J$, so we do not use this filter to constrain the position of component $\mathrm{B}$. The astrometry presented here is the mean of the astrometry derived either in $H+K$ (quasar B) or in $J+H+K$ when the objects are visible in all three bands. The quoted error bars are the errors on this mean value, i.e. the square root of the variance of the different measurements divided by $N$, where $N$ is the number of filters where a measurement is possible.

Flux calibration is carried out using several standard stars, except for the $K_{\mathrm{s}}$-band data. The photometry for all 


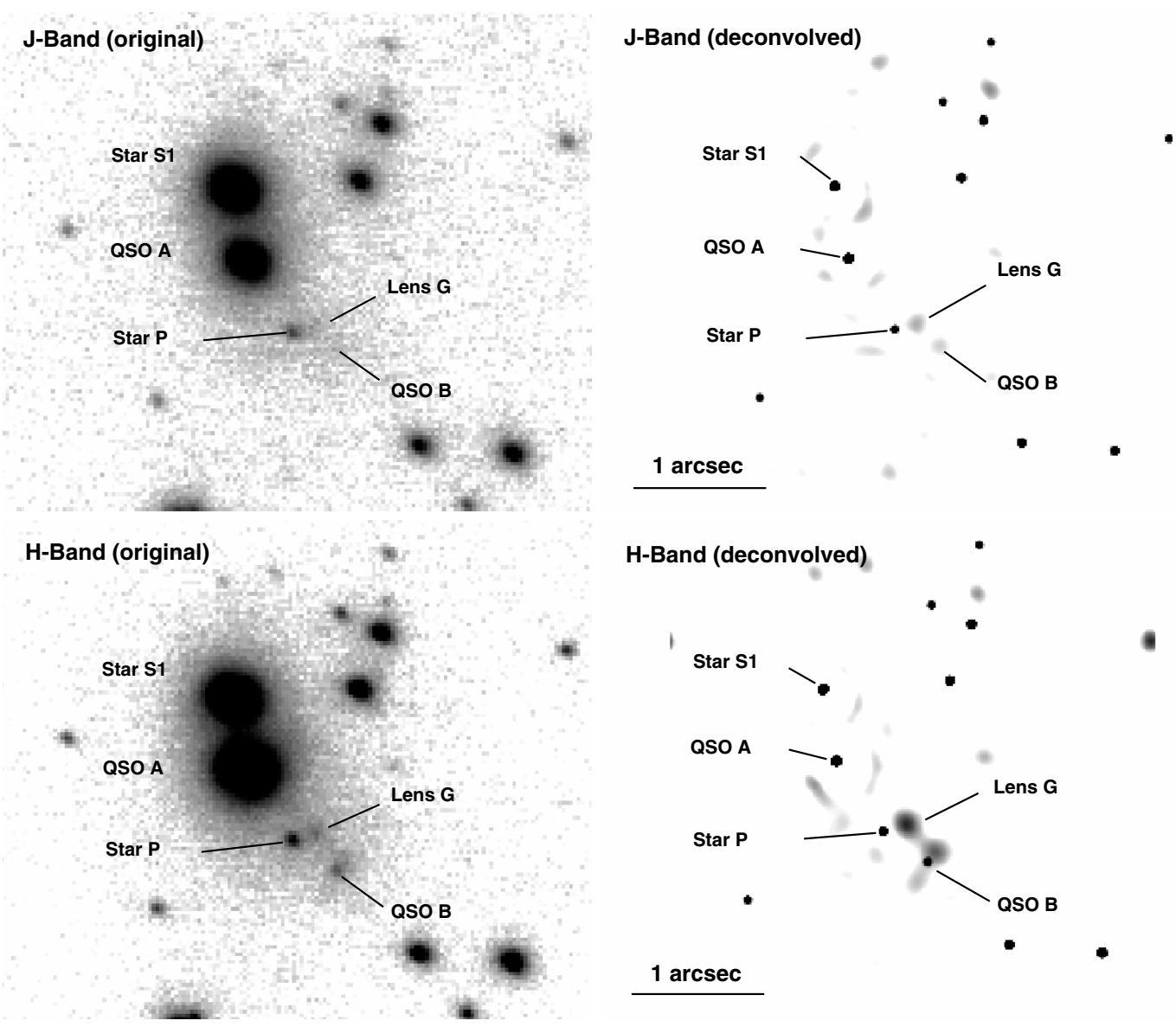

Fig. 2. The combined VLT NACO images of PKS 1830-211 are shown on the left side of the figure, where all objects are labelled as in C02. Lens $\mathrm{G}$ is at the detection limit in the $J$-band, but is clearly detected in $H$. The deconvolved images are shown on the right, with a resolution of $0{ }^{\prime} 027$. In addition to Lens G, an extended object near the Image B of the quasar is seen in the $H$-band deconvolution, and may correspond to the lensed host galaxy of the quasar source. Both in the reduced and deconvolved images, the grey scale displays all intensity levels above $2-\sigma_{\text {sky }}$. Artifacts due to the PSF spatial variations across the CONICA camera, within a radius of $0.5^{\prime \prime}$ around bright point sources are significant, but do not affect Lens $\mathrm{G}$.

Table 2. Astrometry of PKS 1830-211. All positions are given relative to the quasar Image $\mathrm{A}$ along with their 1- $\sigma$ error bars. The measurements have been transformed to match the coordinate system of NICMOS (Lehár et al. 2000), by using 8 stars common to the AO and NICMOS fields of view.

\begin{tabular}{lcc}
\hline \hline Object & $\begin{array}{c}X \\
(\operatorname{arcsec})\end{array}$ & $\begin{array}{c}Y \\
(\operatorname{arcsec})\end{array}$ \\
\hline Quasar A & 0.0 & 0.0 \\
Quasar B & $+0.649 \pm 0.001$ & $-0.724 \pm 0.001$ \\
Star P & $+0.333 \pm 0.002$ & $-0.504 \pm 0.002$ \\
Lens G & $+0.498 \pm 0.004$ & $-0.456 \pm 0.004$ \\
\hline
\end{tabular}

point sources is done by integrating the flux in the analytical Gaussian profiles used to model point sources in the MCS-deconvolved images. Extended objects such as Lens G are measured through 0.2 diameter apertures on the quasarsubtracted images. All photometric measurements are given in Table 3.

We attempt to model Lens $\mathrm{G}$ as a point source when carrying out the MCS deconvolution. The result shows significant residuals in the extended channel of the image, demonstrating that Lens $\mathrm{G}$ is resolved in the AO data. Only the high signalto-noise $H$-band data allow to carry out this experiment. Star P remains unresolved (see Fig. 2), following this test.

\section{Discussion and conclusions}

Our deep high-resolution VLT adaptive optics observations clearly confirm the existence of the second extended object "Lens G". We also confirm that "Star P" is unresolved at the resolution of the present $\mathrm{AO}$ images.

Without any redshift measurement, it is hard to evaluate the relative importance of the two extended objects within the Einstein ring of PKS 1830-211. The only clear clue about a lens redshift in PKS 1830-211 comes from the molecular absorption lines seen in the radio spectrum of the quasar images, suggesting $z_{\text {lens }}=0.89$ (Wiklind \& Combes 1996). This redshift is more likely associated with the spiral lens rather than Lens G. First, the HST images (Fig. 1) show that the arms of the spiral galaxy are passing right in front of the quasar images, especially B. Second, the molecular absorption lines seen in the two quasar images display a velocity difference 


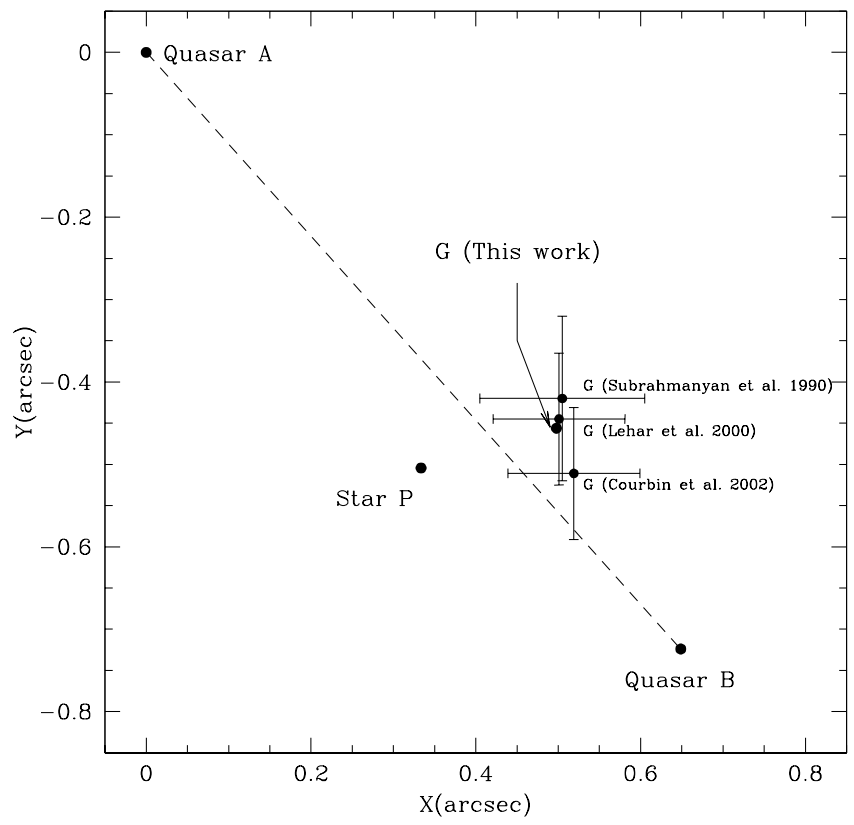

Fig. 3. Summary of the astrometry of PKS 1830-211, relative to the quasar Image A. The position of Lens G is compared with the position found in $\mathrm{C} 02$ and with that of "Object E" seen in the radio by Subrahmanyan et al. (1990).

Table 3. Photometry of the objects in the vicinity of PKS 1830-211, along with their $1-\sigma$ error bars. An additional error bar of 0.05 mag on the photometric zero point is not included here.

\begin{tabular}{lcc}
\hline \hline Object & $J$ & $H$ \\
\hline Quasar A & $18.04 \pm 0.05$ & $16.36 \pm 0.05$ \\
Quasar B & $>23.0$ & $22.12 \pm 0.10$ \\
Star S1 & $17.57 \pm 0.05$ & $16.72 \pm 0.05$ \\
Star P & $21.76 \pm 0.10$ & $20.93 \pm 0.05$ \\
Lens G & $22.76 \pm 0.20$ & $21.25 \pm 0.10$ \\
\hline
\end{tabular}

of $\Delta V=147 \mathrm{~km} \mathrm{~s}^{-1}$, typical of a rotating disk with a small inclination with respect to the plane of the sky. Finally, the $V-I$ color of the spiral $(\mathrm{W} 02, \mathrm{C} 02)$ is indicative a spiral galaxy that has a redshift that is close to $z=1$.

The redshift of Lens $\mathrm{G}$ is much less certain, as photometric data for this object are available only in $J$ and $H$, and with large errors. Lens $\mathrm{G}$ is located only 0 !' 2 away from the center of the spiral disk. Since this is only $1 \mathrm{kpc}$ at $z=0.89$, Lens G could be part of the spiral galaxy seen in the HST images, if both objects are at $z=0.89$.
If Lens $\mathrm{G}$ is not simply part of the spiral galaxy, but is rather the optical counterpart of "Object E" seen in the radio by Subrahmanyan et al. (1990), then the $K$ vs. redshift (or approximately $H$ vs. redshift) relation applies and indicates $z_{\text {lens }}=1.1-1.2$. This redshift is hard to reconcile with lens models. If we take the models by W02 using a single lens centered at the position of Lens G, we obtain values for $\mathrm{H}_{0}$ of about $80 \mathrm{~km} \mathrm{~s}^{-1} \mathrm{Mpc}^{-1}$. Taking into account the fact that W02 compute their models for $z_{\text {lens }}=0.89$ and not $z_{\text {lens }}=1.1-1.2$, the redshift-corrected values for $\mathrm{H}_{0}$ rise above $100 \mathrm{~km} \mathrm{~s}^{-1} \mathrm{Mpc}^{-1}$. Acceptable values for $\mathrm{H}_{0}$, in the range $50<$ $H_{0}<80 \mathrm{~km} \mathrm{~s}^{-1} \mathrm{Mpc}^{-1}$ are found when the redshift of Lens $\mathrm{G}$ is about $z_{\text {lens }}=0.4-0.5$.

Finally, we can not exclude the possibility that Lens $G$ is behind the $z=2.507$ quasar and is not acting as a lens at all. Further study of PKS 1830-211 will require spectroscopy of both Lens $\mathrm{G}$ and the spiral lens, which, given the required spatial resolution and depth, is only possible from space. Spectroscopy of Star P, which could be the bulge of the spiral lens (W02, C02), remains within the reach of large groundbased telescopes with AO.

Acknowledgements. The authors would like to thank Dr. Prasenjit Saha for helpful discussions and the referee, Dr. Joshua Winn, for his very positive and constructive remarks. G.M. and F.C. acknowledge support from the Swiss National Science Foundation (SNSF).

\section{References}

Courbin, F., Lidman, C., Frye, B., et al. 1998, ApJ, 499, L119

Courbin, F., Meylan, G., Kneib, J.-P., \& Lidman, C. 2002, ApJ, 575, 95 (C02)

Jauncey, D. L., Reynolds, J. E., Tzioumis, A. K., et al. 1991, Nature, 352,132

Lehár, J., Falco, E. E., Kochanek, C. S., et al. 2000, ApJ, 536, 584

Lidman, C., Courbin, F., Meylan, G., et al. 1999, ApJ, 514, L57

Magain, P., Courbin, F., \& Sohy, S. 1998, ApJ, 494, 452

Lovell, J. E. J., Jauncey, D. L., Reynolds, J. E., et al. 1998, ApJ, 508, L51

Rao, A. P., \& Subrahmanyan, R. 1988, MNRAS, 231, 229

Subrahmanyan, R., Narasimha, D., Rao, A. P., \& Swarup, G. 1990, MNRAS, 246, 263

Wiklind, T., \& Combes, F. 1996, Nature, 379, 139

Winn, J. N., Kochanek, C. S., McLeod, B. A., et al. 2002, ApJ, 575, 103 (W02) 